\title{
Multiple solutions of semilinear degenerate elliptic boundary value prob- lems
}

\section{Kazuaki Taira ${ }^{1 *}$}

${ }^{1}$ Institute of Mathematics, University of Tsukuba, Tsukuba 305-8571, Japan

Received November 2007

Key words Semilinear elliptic boundary value problem, degenerate boundary condition, the super-sub-solution method, $L^{p}$ Schauder theory MSC (2000) 35J65, 35J25, 47H10

Dedicated to Professor Kyuya Masuda on the occasion of his 70th birthday

The purpose of this paper is to study a class of semilinear elliptic boundary value problems with degenerate boundary conditions which include as particular cases the Dirichlet problem and the Robin problem. The approach here is based on the super-sub-solution method in the degenerate case, and is distinguished by the extensive use of an $L^{p}$ Schauder theory elaborated for second-order, elliptic differential operators with discontinuous zero-th order term. By using Schauder's fixed point theorem, we prove that the existence of an ordered pair of sub- and supersolutions of our problem implies the existence of a solution of the problem. The results extend an earlier theorem due to Kazdan and Warner to the degenerate case.

Copyright line will be provided by the publisher

\section{Statement of main results}

Let $\Omega$ be a bounded domain of Euclidean space $\mathbf{R}^{N}, N \geq 2$, with smooth boundary $\partial \Omega$; its closure $\bar{\Omega}=\Omega \cup \partial \Omega$ is an $N$ dimensional, compact smooth manifold with boundary. Let $A$ be a second-order, elliptic differential operator with real coefficients such that

$$
A u:=-\sum_{i=1}^{N} \frac{\partial}{\partial x_{i}}\left(\sum_{j=1}^{N} a^{i j}(x) \frac{\partial u}{\partial x_{j}}\right)+c(x) u .
$$

Here:

(1) $a^{i j} \in C^{\infty}(\bar{\Omega})$ and $a^{i j}(x)=a^{j i}(x)$ on $\bar{\Omega}$, and there exists a positive constant $a_{0}$ such that

$$
\sum_{i, j=1}^{N} a^{i j}(x) \xi_{i} \xi_{j} \geq a_{0}|\xi|^{2}, \quad x \in \bar{\Omega}, \xi=\left(\xi_{1}, \xi_{2}, \ldots, \xi_{N}\right) \in \mathbf{R}^{N}
$$

(2) The function $c(x)$ is real-valued and may be discontinuous in $\Omega$. More precisely, $c \in L^{\infty}(\Omega)$ and $c(x) \geq 0$ almost everywhere in $\Omega$.

Let $B$ be a first-order, boundary condition with real coefficients such that

$$
B u:=a\left(x^{\prime}\right) \frac{\partial u}{\partial \nu}+b\left(x^{\prime}\right) u \quad \text { on } \partial \Omega .
$$

Here:

(3) $a \in C^{\infty}(\partial \Omega)$ and $a\left(x^{\prime}\right) \geq 0$ on $\partial \Omega$.

\footnotetext{
* E-mail: taira@math.tsukuba.ac.jp
} 
(4) $b \in C^{\infty}(\partial \Omega)$ and $b\left(x^{\prime}\right) \geq 0$ on $\partial \Omega$.

(5) $\partial / \partial \boldsymbol{\nu}$ is the conormal derivative associated with the operator $A$,

$$
\frac{\partial}{\partial \boldsymbol{\nu}}=\sum_{i, j=1}^{N} a^{i j}\left(x^{\prime}\right) n_{j} \frac{\partial}{\partial x_{i}}
$$

where $\mathbf{n}=\left(n_{1}, n_{2}, \ldots, n_{N}\right)$ is the unit exterior normal to the boundary $\partial \Omega$.

We remark that the boundary condition $B$ given by formula (1.2) is a smooth linear combination of the Dirichlet condition and the Neumann condition.

In this paper we consider the following semilinear elliptic boundary value problem: Given a function $f(x, \xi)$ defined on $\bar{\Omega} \times \mathbf{R}$, find a function $u(x)$ in $\Omega$ such that

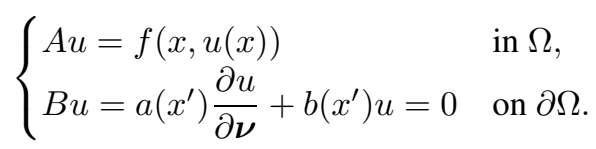

Our fundamental hypotheses on the boundary condition $B$ are the following:

(H.1) $a\left(x^{\prime}\right)+b\left(x^{\prime}\right)>0$ on $\partial \Omega$.

(H.2) $b\left(x^{\prime}\right) \not \equiv 0$ on $\partial \Omega$.

It should be noticed that if $a\left(x^{\prime}\right) \equiv 0$ and $b\left(x^{\prime}\right) \equiv 1$ on $\partial \Omega$ (resp. $a\left(x^{\prime}\right)>0$ on $\partial \Omega$ ), then the boundary condition $B$ is the Dirichlet condition (resp. Robin condition). It is easy to see that problem (1.3) is nondegenerate (or coercive) if and only if either $a\left(x^{\prime}\right)>0$ on $\partial \Omega$ or $a\left(x^{\prime}\right) \equiv 0$ and $b\left(x^{\prime}\right)>0$ on $\partial \Omega$. Amann [2] and Amann-Crandall [3] studied problem (1.3) under the condition that the boundary $\partial \Omega$ is the disjoint union of the two closed subsets $M=\left\{x^{\prime} \in \partial \Omega: a\left(x^{\prime}\right)=0\right\}$ and $\partial \Omega \backslash M=\left\{x^{\prime} \in \partial \Omega: a\left(x^{\prime}\right)>0\right\}$, each of which is an $(N-1)$ dimensional compact smooth manifold. What is the important feature of our boundary condition $B$ is that problem (1.3) becomes a degenerate boundary value problem from an analytical point of view. This is due to the fact that, having a first order pseudo-differential operator on the boundary $\partial \Omega$, the so-called Shapiro-Lopatinskii complementary condition is violated at each point of the set $M$ (cf. [8]).

We remark that problem (1.3) is closely related to population dynamics in environments with spatial heterogeneity (cf. [16], [17]). In terms of biology, the functions $a\left(x^{\prime}\right)$ and $b\left(x^{\prime}\right)$ measure the hostility of the exterior of the domain. For example, if $a\left(x^{\prime}\right) \equiv 0$ and $b\left(x^{\prime}\right) \equiv 1$ on $\partial \Omega$, then the Dirichlet condition $B$ represents that $\Omega$ is surrounded by a completely hostile exterior such that any member of the population which reaches the boundary dies immediately; in other words, the exterior of the domain is deadly to the population. If $a\left(x^{\prime}\right) \equiv 1$ and $b\left(x^{\prime}\right) \equiv 0$ on $\partial \Omega$, then the Neumann condition $B$ represents that the boundary acts as a barrier, that is, individuals reaching the boundary simply return to the interior. The intuitive meaning of hypothesis (H.1) implies that individuals reaching the set $M$ die immediately. Namely, the boundary portion $M$ is deadly to the population. On the other hand, hypothesis (H.2) implies that the boundary $\partial \Omega$ is not a complete barrier.

Our approach to problem (1.3) is based on the super-sub-solution method which goes back to Sattinger [13] in the non-degenerate case. However, it should be emphasized that, in the case where $c \in L^{\infty}(\Omega)$, the classical Schauder theory breaks down for problem (1.3). In fact, the solutions of problem (1.3) are not necessarily smooth on $\bar{\Omega}$. Taira [15] and Runst [10] and Runst-Il'yasov [11] studied problem (1.3) under the condition that $c \in C^{\infty}(\bar{\Omega})$ (see also [7, Chapter 33] for the abstract setting). The crucial point in our approach is how to control the discontinuous function $c(x)$ in formula (1.1) in terms of Sobolev spaces of $L^{p}$ type, just as in Taira [17].

In order to study problem (1.3) in the framework of Sobolev spaces of $L^{p}$ type, we impose the following regularity condition (F.1) on the nonlinear term $f(x, \xi)$ :

(F.1) The function $f(x, \xi)$ is real-valued and continuous on $\bar{\Omega} \times \mathbf{R}$, and its partial derivative $\partial f / \partial \xi$ exists and is continuous on $\bar{\Omega} \times \mathbf{R}$. 
A function $u \in W^{2, p}(\Omega), N<p<\infty$, is called a solution of problem (1.3) if it satisfies the conditions

$$
\begin{cases}A u=f(x, u(x)) & \text { almost everywhere in } \Omega, \\ B u=0 & \text { on } \partial \Omega .\end{cases}
$$

Here it should be noticed that we have, by Sobolev's imbedding theorem (see [1, Theorem 4.12]),

$$
W^{2, p}(\Omega) \subset C^{1}(\bar{\Omega}), \quad N<p<\infty .
$$

A function $\psi \in W^{2, p}(\Omega), N<p<\infty$, is called a supersolution of problem (1.3) if it satisfies the conditions

$$
\begin{cases}A \psi \geq f(x, \psi(x)) & \text { almost everywhere in } \Omega, \\ B \psi \geq 0 & \text { on } \partial \Omega .\end{cases}
$$

Similarly, a function $\phi \in W^{2, p}(\Omega), N<p<\infty$, is called a subsolution of problem (1.3) if it satisfies the conditions

$$
\begin{cases}A \phi \leq f(x, \phi(x)) & \text { almost everywhere in } \Omega, \\ B \phi \leq 0 & \text { on } \partial \Omega .\end{cases}
$$

Now we are in a position to state our fundamental existence theorem of solutions of problem (1.3). The next theorem asserts that the existence of an ordered pair of sub- and supersolutions implies the existence of a solution of problem (1.3) (see [2, Theorem 9.4] in the non-degenerate case):

Theorem 1.1 Assume that the nonlinear term $f(x, \xi)$ satisfies the regularity condition (F.1). If $\psi(x)$ and $\phi(x)$ are respectively super- and subsolutions of problem (1.3) which satisfy the condition that $\phi(x) \leq \psi(x)$ on $\bar{\Omega}$, then there exists a solution $u \in W^{2, p}(\Omega), N<p<\infty$, of problem (1.3) such that $\phi(x) \leq u(x) \leq \psi(x)$ on $\bar{\Omega}$.

Remark 1.2 Taira [14] considered the smooth case where

$$
c \in C^{\infty}(\bar{\Omega}) \text { and } c(x)>0 \text { in } \Omega,
$$

and proved Theorem 1.1 under the conditions that the nonlinear term $f(x, \xi, \eta)$ is a $C^{1}$ function on $\bar{\Omega} \times \mathbf{R} \times \mathbf{R}^{N}$ and that there exists a non-negative and increasing function $d(r)$ on the interval $[0, \infty)$ such that

$$
|f(x, \xi, \eta)| \leq d(|\xi|)\left(1+|\eta|^{2}\right), \quad(x, \xi, \eta) \in \bar{\Omega} \times \mathbf{R} \times \mathbf{R}^{N} .
$$

As an application of Theorem 1.1, we give various sufficient conditions for a solution of problem (1.3) to exist. To do this, we study the linearized elliptic boundary value problem

$$
\begin{cases}A u=g & \text { in } \Omega, \\ B u=0 & \text { on } \partial \Omega\end{cases}
$$

in the framework of the Hilbert space $L^{2}(\Omega)$. We associate with problem (1.4) a densely defined, closed linear operator

$$
\mathfrak{A}: L^{2}(\Omega) \longrightarrow L^{2}(\Omega)
$$

as follows:

(1) $\mathcal{D}(\mathfrak{A}):=\left\{u \in W^{2,2}(\Omega): B u=0\right.$ on $\left.\partial \Omega\right\}$.

(2) $\mathfrak{A} u:=A u, u \in \mathcal{D}(\mathfrak{A})$.

Then we have the following three fundamental results (i), (ii) and (iii) (see [17, Theorem 5.1]):

(i) The operator $\mathfrak{A}$ is positive and selfadjoint in $L^{2}(\Omega)$. 
(ii) The first eigenvalue $\lambda_{1}$ of $\mathfrak{A}$ is positive and algebraically simple, and the corresponding eigenfunction $\varphi_{1} \in$ $W^{2, p}(\Omega), N<p<\infty$, may be chosen to be strictly positive in $\Omega$. Namely, we have the assertions

$$
\begin{cases}A \varphi_{1}=\lambda_{1} \varphi_{1} & \text { in } \Omega \\ \varphi_{1}>0 & \text { in } \Omega \\ B \varphi_{1}=0 & \text { on } \partial \Omega\end{cases}
$$

(iii) No other eigenvalues $\lambda_{j}, j \geq 2$, have positive eigenfunctions.

The next existence theorem of solutions of problem (1.3) is a generalization of Kazdan-Warner [9, Corollary 2.12] to the degenerate case (cf. [12, Theorem 6.5.2/2]):

Theorem 1.3 Assume that the nonlinear term $f(x, \xi)$ satisfies the regularity condition (F.1) and one of the following three conditions $(A),(B)$ and $(C)$ :

(A) There exists a continuous function $h(x)$ on $\bar{\Omega}$ such that

$$
\begin{aligned}
& \int_{\Omega} h(x) \varphi_{1}(x) d x<0, \\
& \limsup _{|\xi| \rightarrow \infty} \frac{\xi}{|\xi|}\left[f(x, \xi)-\lambda_{1} \xi\right]<h(x), \quad x \in \bar{\Omega},
\end{aligned}
$$

where $\lim \sup _{|\xi| \rightarrow \infty}$ is assumed to be uniform for $x \in \bar{\Omega}$.

(B) We have the condition

$$
\limsup _{|\xi| \rightarrow \infty} \frac{f(x, \xi)}{\xi}<\lambda_{1}
$$

where $\lim \sup _{|\xi| \rightarrow \infty}$ is assumed to be uniform for $x \in \bar{\Omega}$.

(C) We have the condition

$$
\limsup _{|\xi| \rightarrow \infty} \frac{\partial f}{\partial \xi}(x, \xi)<\lambda_{1},
$$

where $\lim \sup _{|\xi| \rightarrow \infty}$ is assumed to be uniform for $x \in \bar{\Omega}$.

Then the semilinear problem (1.3) has at least one solution $u \in W^{2, p}(\Omega)$ with $N<p<\infty$.

Example 1.4 A simple example of the nonlinear term $f(x, \xi)$ is given by the formula

$$
f(x, \xi)= \begin{cases}\gamma\left(\xi+\frac{1}{2 \xi}-\frac{5}{4}\right) & \text { for } \xi>1, \\ \frac{\gamma}{4} \xi^{2} & \text { for }-1 \leq \xi \leq 1, \\ \gamma\left(-\xi-\frac{1}{2 \xi}-\frac{5}{4}\right) & \text { for } \xi<-1\end{cases}
$$

where $\gamma$ is a constant such that $0<\gamma<\lambda_{1}$. It is easy to verify that the function $f(x, \xi)$ satisfies condition (F.1) and condition $(\mathrm{C})$.

The essential point in the proof of Theorem 1.3 is to reduce the study of the semilinear problem (1.3) to that of the semilinear problem with linear part at resonance:

$$
\begin{cases}A u-\lambda_{1} u=f(x, u(x)) & \text { in } \Omega \\ B u=a\left(x^{\prime}\right) \frac{\partial u}{\partial \nu}+b\left(x^{\prime}\right) u=0 & \text { on } \partial \Omega .\end{cases}
$$


Moreover, we consider the following semilinear elliptic boundary value problem depending on a parameter: Given a function $F(x, \xi, t)$ defined on $\bar{\Omega} \times \mathbf{R} \times \mathbf{R}$, find a function $u(x)$ in $\Omega$ such that

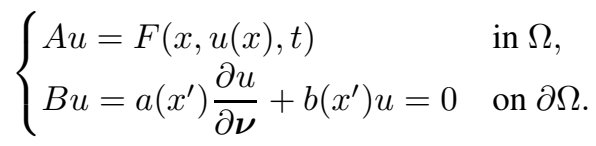

The parameter $t$ may enter naturally in a manner similar to an eigenvalue. In order to state our existence theorem of solutions of problem (1.5), we impose the following regularity condition (F.2) on the nonlinear term $F(x, \xi, t)$ :

(F.2) The function $F(x, \xi, t)$ is real-valued and continuous on $\bar{\Omega} \times \mathbf{R} \times \mathbf{R}$, and its partial derivatives $\partial F / \partial \xi$ and $\partial F / \partial t$ exist and are continuous on $\bar{\Omega} \times \mathbf{R} \times \mathbf{R}$.

The next existence theorem of solutions of problem (1.5) extends Kazdan-Warner [9, Corollary 3.11] to the degenerate case (cf. [12, Theorem 6.5.3/1]):

Theorem 1.5 Assume that the nonlinear term $F(x, \xi, t)$ satisfies the regularity condition (F.2) and further that the following three conditions (D.1), (D.2) and (D.3) are satisfied:

(D.1) For every $m \in \mathbf{R}$, there exists a continuous function $h_{m}(x)$ on $\bar{\Omega}$, depending on $m$, such that

$$
\frac{\partial F}{\partial t}(x, \xi, t) \geq h_{m}(x)>0, \quad x \in \Omega, \xi \geq m, t \in \mathbf{R} .
$$

(D.2) We have, for every $t \in \mathbf{R}$,

$$
\limsup _{\xi \rightarrow-\infty} \frac{F(x, \xi, t)}{\xi}<\lambda_{1}
$$

where $\lim \sup _{\xi \rightarrow-\infty}$ is assumed to be uniform for $x \in \bar{\Omega}$.

(D.3) There exist constants $t_{1}, m_{1}$ and $c>0$ such that

$$
F\left(x, \xi, t_{1}\right)-\lambda_{1} \xi>c, \quad x \in \bar{\Omega}, \xi>m_{1} .
$$

Then there exists a number $t_{0} \in \mathbf{R}$ such that the semilinear problem (1.5) has at least one solution $u \in W^{2, p}(\Omega)$, $N<p<\infty$, for $t<t_{0}$, but it has no solution for $t>t_{0}$.

Example 1.6 A simple example of the nonlinear term $F(x, \xi, t)$ is given by the formula

$$
F(x, \xi, t)=\operatorname{tr}(x) e^{\xi}+p(\xi) .
$$

Here:

(1) $r \in C(\bar{\Omega})$ and $r(x)>0$ in $\Omega$.

(2) $p(\xi)$ is a real-valued, $C^{1}$ function defined by the formula

$$
p(\xi)= \begin{cases}\gamma^{\prime \prime} \xi+C_{1} \frac{\gamma^{\prime \prime}}{\xi}+C_{2} & \text { for } \xi>1 \\ C_{3} \xi^{3}+C_{4} \xi(\xi+1) & \text { for }-1 \leq \xi \leq 1 \\ \gamma^{\prime} \xi+\frac{\gamma^{\prime}}{2 \xi}+C_{5} & \text { for } \xi<-1,\end{cases}
$$

where $0<\gamma^{\prime}<\lambda_{1}, \gamma^{\prime \prime}>\lambda_{1}$ and the $C_{i}$ are some suitable constants.

It is easy to verify that the function $F(x, \xi, t)$ satisfies condition (F.2) and conditions (D.1), (D.2) and (D.3).

Finally, the next corollary extends Kazdan-Warner [9, Corollary 3.12] to the degenerate case (cf. [12, Theorem 6.5.3/3]): 
Corollary 1.7 Let the nonlinear term $F(x, \xi, t)$ be of the special form

$$
F(x, \xi, t)=f_{0}(x)+\operatorname{tr}(x)+g(x, \xi), \quad(x, \xi, t) \in \bar{\Omega} \times \mathbf{R} \times \mathbf{R},
$$

where $f_{0} \in C(\bar{\Omega}), r \in C(\bar{\Omega})$ with $r(x)>0$ in $\Omega$ and $g \in C^{1}(\bar{\Omega} \times \mathbf{R})$. We assume that the following two conditions (E.1) and (E.2) are satisfied:

(E.1) We have the condition

$$
\limsup _{\xi \rightarrow-\infty} \frac{g(x, \xi)}{\xi}<\lambda_{1}
$$

where $\lim \sup _{\xi \rightarrow-\infty}$ is assumed to be uniform for $x \in \bar{\Omega}$.

(E.2) There exist constants $m_{1}$ and $k$ such that

$$
g(x, \xi)-\lambda_{1} \xi>k, \quad x \in \bar{\Omega}, \xi>m_{1} .
$$

Then there exists a number $t_{0} \in \mathbf{R}$ such that the semilinear elliptic boundary value problem

$$
\begin{cases}A u=f_{0}(x)+\operatorname{tr}(x)+g(x, u(x)) & \text { in } \Omega, \\ B u=0 & \text { on } \partial \Omega\end{cases}
$$

has at least one solution $u \in W^{2, p}(\Omega), N<p<\infty$, for $t<t_{0}$, but it has no solution for $t>t_{0}$.

The rest of this paper is organized as follows. In Section 2, by using Schauder's fixed point theorem we prove Theorem 1.1. Namely, we prove that the existence of an ordered pair of sub- and supersolutions of problem (1.3) implies the existence of a solution of problem (1.3). Section 3 is devoted to the proof of Theorem 1.3. In Subsection 3.1 we make use of the Lyapunov-Schmidt procedure to construct sub- and supersolutions of problem (1.3), and then apply Theorem 1.1. This subsection is the heart of the subject. The essential step in the proof of Theorem 1.3 is Lemma 3.2 in which we construct a supersolution of problem (1.3) provided that the nonlinear term $f(x, \xi)$ satisfies condition $\left(A_{+}\right)$. The crucial point is how to adjust the classical method to the case where $c \in L^{\infty}(\Omega)$, by using an $L^{p}$ Schauder theory. In Section 4 we prove Theorem 1.5 and Corollary 1.7. Our proof is based on the super-sub-solution method, just as in the proof of Theorem 1.3. To do this, we use a parameter-dependent version of Theorem 1.1 (Theorem 4.1). The essential step in the proof of Theorem 1.5 is Theorem 4.3 which gives sufficient conditions in order that $t_{0}>-\infty$. In the final Section 5, as an application of Theorem 4.1 we obtain the existence theorem of positive solutions of the semilinear elliptic eigenvalue problem (5.1) (Theorem 5.1) which extends Kazdan-Warner [9, Proposition 4.2] to the degenerate case. The approach here is distinguished by the extensive use of the ideas and techniques characteristic of the recent developments in the theory of partial differential equations (see [17]).

\section{Proof of Theorem 1.1}

In this section, by using Schauder's fixed point theorem we prove Theorem 1.1. Our proof of Theorem 1.1 is based on a variant of the Bakel'man and Aleksandrov maximum principle in the framework of Sobolev spaces due to Bony [4] (see also [18]), just as in Taira [17, Proposition 3.12]. The proof is divided into three steps.

Step 1: First, by the regularity condition (F.1) it follows that the nonlinear term $f(x, \xi)$ satisfies the slope condition. Namely, for any given positive number $\sigma$, there exists a positive constant $\omega=\omega(\sigma)$, independent of $x \in \bar{\Omega}$, such that

$$
f(x, \xi)-f(x, \eta)>-\omega \cdot(\xi-\eta), \quad x \in \bar{\Omega},-\sigma \leq \eta<\xi \leq \sigma .
$$

Geometrically, this condition means that the slope of the function $\xi \mapsto f(x, \xi)$ is bounded below, uniformly with respect to $x \in \bar{\Omega}$. 
First, we replace the function $c(x)$ by the function $c(x)+\omega$, where $\omega>0$ is the same constant as in condition (2.1), and consider instead of problem (1.3) the following problem:

$$
\begin{cases}(A+\omega) u=\omega u+F(u) & \text { in } \Omega, \\ B u=a\left(x^{\prime}\right) \frac{\partial u}{\partial \boldsymbol{\nu}}+b\left(x^{\prime}\right) u=0 & \text { on } \partial \Omega,\end{cases}
$$

where $F$ is the Nemytskii operator of $f$ defined by the formula

$$
F u(x)=f(x, u(x)), \quad x \in \bar{\Omega} .
$$

By applying [17, Theorem 1.1] to our situation, we find that the boundary value problem

$$
\begin{cases}(A+\omega) v=g & \text { in } \Omega \\ B v=0 & \text { on } \partial \Omega\end{cases}
$$

has a unique solution $v \in W^{2, p}(\Omega)$ for any function $g \in L^{p}(\Omega)$. Hence, we can introduce a continuous linear operator (resolvent) $K_{\omega}: L^{p}(\Omega) \rightarrow W^{2, p}(\Omega)$ by the formula $v=K_{\omega} g$. Then it is clear that problem (1.3) is equivalent to problem (2.2). Furthermore, since $f(x, \xi)$ is continuous on $\bar{\Omega} \times \mathbf{R}$, we obtain that problem (2.2) is equivalent to an operator equation

$$
u=K_{\omega}(\omega u+F(u)) \quad \text { in } C(\bar{\Omega}) .
$$

Step 2: We let

$$
H_{\omega}(u):=K_{\omega}(\omega u+F(u)), \quad u \in C(\bar{\Omega}) .
$$

The next lemma asserts that the map $H_{\omega}(\cdot)$ leaves invariant the ordering of the ordered Banach space $C(\bar{\Omega})$ (see [15, Lemma 4.1]):

Lemma 2.1 The operator $H_{\omega}(\cdot):[\phi, \psi] \rightarrow C(\bar{\Omega})$ is increasing. Here $[\phi, \psi]$ is the order interval defined by the formula

$$
[\phi, \psi]=\{u \in C(\bar{\Omega}): \phi(x) \leq u(x) \leq \psi(x) \text { on } \bar{\Omega}\} .
$$

Moreover, we have the following (see [15, Lemma 4.2]):

Lemma 2.2 The operator $H_{\omega}(\cdot)=K_{\omega}(\omega \cdot+F(\cdot))$ maps the order interval $[\phi, \psi]$ into itself.

Step 3: It should be noticed that we have, by Sobolev's imbedding theorem (see [1, Theorem 4.12]),

$$
W^{2, p}(\Omega) \subset C^{2-N / p}(\bar{\Omega}), \quad N<p<\infty .
$$

Hence it follows from an application of the Ascoli-Arzelà theorem that the resolvent $K_{\omega}$, considered as an operator $K_{\omega}: C(\bar{\Omega}) \rightarrow C^{1}(\bar{\Omega})$, is compact and further from an application of Lemma 2.2 that the mapping $H_{\omega}(\cdot):[\phi, \psi] \rightarrow[\phi, \psi]$ is compact. We remark that the order interval $[\phi, \psi]$ is closed, bounded and convex in the space $C(\bar{\Omega})$. Therefore, by applying Schauder's fixed point theorem (see [19, Theorem 2.A]; [5, Corollary 2.3.10]) to our situation we can find a solution $u \in[\phi, \psi]$ of equation (2.3).

The proof of Theorem 1.1 is complete.

\section{Proof of Theorem 1.3}

In this section, by using Theorem 1.1 we prove our existence theorem of solutions of problem (1.3) (Theorem 1.3). To do this, we construct sub- and supersolutions of problem (1.3) in Subsection 3.1 by means of the LyapunovSchmidt procedure. The essential step in the proof of Theorem 1.3 is Lemma 3.2 in which we construct a supersolution of problem (1.3) provided that the nonlinear term $f(x, \xi)$ satisfies condition $\left(A_{+}\right)$. 


\subsection{Construction of sub- and supersolutions}

In this subsection we give useful conditions in finding subsolutions and supersolutions of problem (1.3).

First, we introduce the following four conditions $\left(A_{ \pm}\right)$and $\left(B_{ \pm}\right)$on the nonlinear term $f(x, \xi)$ :

$\left(A_{+}\right)$There exist a number $s_{+} \in \mathbf{R}$ and a bounded continuous function $g_{+}(x, \xi)$ on $\bar{\Omega} \times \mathbf{R}$ such that if $u(x)>$ $s_{+} \varphi_{1}(x)$ in $\Omega$, then we have the inequalities

$$
\begin{aligned}
& f(x, u(x)) \leq g_{+}(x, u(x)), \quad x \in \Omega, \\
& \int_{\Omega} g_{+}(x, u(x)) \varphi_{1}(x) d x \leq 0 .
\end{aligned}
$$

$\left(A_{-}\right)$There exist a number $s_{-} \in \mathbf{R}$ and a bounded continuous function $g_{-}(x, \xi)$ on $\bar{\Omega} \times \mathbf{R}$ such that if $u(x)<$ $s_{-} \varphi_{1}(x)$ in $\Omega$, then we have the inequalities

$$
\begin{aligned}
& f(x, u(x)) \geq g_{-}(x, u(x)), \quad x \in \Omega, \\
& \int_{\Omega} g_{-}(x, u(x)) \varphi_{1}(x) d x \geq 0 .
\end{aligned}
$$

$\left(B_{+}\right)$There exists a continuous function $h_{+}(x)$ on $\bar{\Omega}$ such that

$$
\begin{aligned}
& \int_{\Omega} h_{+}(x) \varphi_{1}(x) d x<0, \\
& \limsup _{\xi \rightarrow+\infty} f(x, \xi)<h_{+}(x), \quad x \in \bar{\Omega},
\end{aligned}
$$

where $\lim \sup _{\xi \rightarrow+\infty}$ is assumed to be uniform for $x \in \bar{\Omega}$.

$\left(B_{-}\right)$There exists a continuous function $h_{-}(x)$ on $\bar{\Omega}$ such that

$$
\begin{aligned}
& \int_{\Omega} h_{-}(x) \varphi_{1}(x) d x>0, \\
& \liminf _{\xi \rightarrow-\infty} f(x, \xi)>h_{-}(x), \quad x \in \bar{\Omega},
\end{aligned}
$$

where $\liminf _{\xi \rightarrow-\infty}$ is assumed to be uniform for $x \in \bar{\Omega}$.

Then we have the following (see [9, Lemma 2.3]):

Lemma 3.1 If the function $f(x, \xi)$ satisfies condition $\left(B_{+}\right)$, then it satisfies condition $\left(A_{+}\right)$. Similarly, if the function $f(x, \xi)$ satisfies condition $\left(B_{-}\right)$, then it satisfies condition $\left(A_{-}\right)$.

In order to prove Theorem 1.3, we consider the following semilinear elliptic boundary value problem with linear part at resonance:

$$
\begin{cases}A u-\lambda_{1} u=f(x, u(x)) & \text { in } \Omega, \\ B u=a\left(x^{\prime}\right) \frac{\partial u}{\partial \boldsymbol{\nu}}+b\left(x^{\prime}\right) u=0 & \text { on } \partial \Omega .\end{cases}
$$

The next lemma is an essential step in the proof of Theorem 1.3 (cf. [9, Lemma 2.7]):

Lemma 3.2 Assume that a function $z \in W^{2, p}(\Omega), N<p<\infty$, satisfies the boundary condition

$$
B z=a\left(x^{\prime}\right) \frac{\partial z}{\partial \nu}+b\left(x^{\prime}\right) z=0 \quad \text { on } \partial \Omega .
$$

If the function $f(x, \xi)$ satisfies condition $\left(A_{+}\right)$, then we can construct a supersolution $u_{+} \in W^{2, p}(\Omega)$ of problem (3.1) which satisfies the condition

$$
u_{+}(x)>z(x) \text { in } \Omega \text {. }
$$


Similarly, if the function $f(x, \xi)$ satisfies condition $\left(A_{-}\right)$, then we can construct a subsolution $u_{-} \in W^{2, p}(\Omega)$ of problem (3.1) which satisfies the condition

$$
u_{-}(x)<z(x) \text { in } \Omega \text {. }
$$

Proof. The proof of Lemma 3.2 is based on the Lyapunov-Schmidt procedure which reduces an infinitedimensional problem to a finite-dimensional system. The proof is divided into three steps.

Step 1: First, we remark the following orthogonal decomposition of $L^{p}(\Omega)$ in the Hilbert space $L^{2}(\Omega)$ :

$$
\begin{aligned}
L^{p}(\Omega) & =\left\{u \in L^{p}(\Omega):\left(A-\lambda_{1}\right) u=0, B u=0\right\} \oplus\left\{\left(A-\lambda_{1}\right) u: u \in W^{2, p}(\Omega), B u=0\right\} \\
& :=\mathcal{N}\left(A_{p}-\lambda_{1} I\right) \oplus \mathcal{R}\left(A_{p}-\lambda_{1} I\right) \\
& =\operatorname{span}\left[\varphi_{1}\right] \oplus \mathcal{R}\left(A_{p}-\lambda_{1} I\right)
\end{aligned}
$$

where $A_{p}: L^{p}(\Omega) \rightarrow L^{p}(\Omega)$ is a densely defined, closed linear operator defined as follows:

(1) $\mathcal{D}\left(A_{p}\right):=\left\{u \in W^{2, p}(\Omega): B u=0\right.$ on $\left.\partial \Omega\right\}$.

(2) $A_{p} u:=A u, u \in \mathcal{D}\left(A_{p}\right)$.

Indeed, it suffices to note the following three assertions (i), (ii), and (iii):

(i) Since the operator $A_{2}=\mathfrak{A}$ is selfadjoint in $L^{2}(\Omega)$, it follows that

$$
\begin{aligned}
& L^{2}(\Omega)=\mathcal{N}\left(A_{2}-\lambda_{1} I\right) \oplus \mathcal{R}\left(A_{2}-\lambda_{1} I\right), \\
& \operatorname{codim} \mathcal{R}\left(A_{2}-\lambda_{1} I\right)=\operatorname{dim} \mathcal{N}\left(A_{2}-\lambda_{1} I\right) .
\end{aligned}
$$

(ii) The null space $\mathcal{N}\left(A_{p}-\lambda_{1} I\right)$ does not depend on $p$ and $\mathcal{N}\left(A_{p}-\lambda_{1} I\right)=\mathcal{N}\left(\mathfrak{A}-\lambda_{1} I\right)=\operatorname{span}\left[\varphi_{1}\right]$ for all $p>1$ (see [17, Theorem 3.3]).

(iii) If $f \in L^{p}(\Omega)$ for $N<p<\infty$ and if $u \in \mathcal{D}\left(A_{2}\right)$ satisfies the equation $\left(A_{2}-\lambda_{1} I\right) u=f$, then it follows that $u \in W^{2, p}(\Omega)$, that is, $u \in \mathcal{D}\left(A_{p}\right)$ (see [17, Theorem 1.1]).

Now we assume that the function $f(x, \xi)$ satisfies condition $\left(A_{+}\right)$. If $w \in L^{p}(\Omega)$, then we consider the function

$$
\begin{aligned}
& G(x, w(x)) \\
:= & g_{+}(x, w(x))-\left(\int_{\Omega} g_{+}(y, w(y)) \varphi_{1}(y) d y\right) \varphi_{1}(x) \in \mathcal{R}\left(A_{p}-\lambda_{1} I\right)=\mathcal{N}\left(A_{p}-\lambda_{1} I\right)^{\perp} .
\end{aligned}
$$

Then we have, for some positive constant $k$,

$$
|G(x, w(x))| \leq k, \quad x \in \Omega,
$$

since the function $g_{+}(x, \xi)$ is bounded. Hence we can find a unique solution $u \in W^{2, p}(\Omega) \cap \mathcal{R}\left(A_{p}-\lambda_{1}\right)$ of the linear problem

$$
\begin{cases}A u-\lambda_{1} u=G(x, w(x)) & \text { in } \Omega, \\ B u=0 & \text { on } \partial \Omega .\end{cases}
$$

Since $u \in \mathcal{D}\left(A_{p}\right) \cap \mathcal{R}\left(A_{p}-\lambda_{1}\right)$, by decomposition (3.4) and [17, Theorem 1.1] we have the a priori estimate

$$
\|u\|_{W^{2, p}(\Omega)} \leq C\left\|A u-\lambda_{1} u\right\|_{L^{p}(\Omega)}=C\|G(\cdot, w)\|_{L^{p}(\Omega)}, \quad u \in W^{2, p}(\Omega) \cap \mathcal{R}\left(A_{p}-\lambda_{1}\right),
$$

where $C$ is a positive constant independent of $u$ and $w$.

Therefore, by combining estimates (3.5) and (3.7) we obtain that

$$
\|u\|_{W^{2, p}(\Omega)}=\left\|\left(A-\lambda_{1} I\right)^{-1}(G(\cdot, w))\right\|_{W^{2, p}(\Omega)} \leq C\|G(\cdot, w)\|_{L^{p}(\Omega)} \leq k C|\Omega|^{1 / p},
$$


where $|\Omega|$ denotes the volume of $\Omega$. However, we have, by Sobolev's imbedding theorem,

$$
u \in W^{2, p}(\Omega) \subset C^{1}(\Omega) \subset W^{1, \infty}(\Omega), \quad N<p<\infty,
$$

and so, for some positive constant $C_{0}$,

$$
\|u\|_{W^{1, \infty}(\Omega)} \leq C_{0}\|u\|_{W^{2, p}(\Omega)} \leq k C_{0} C|\Omega|^{1 / p} .
$$

This proves that any solution $u(x)$ of problem (3.6) is uniformly bounded in the Sobolev space $W^{1, \infty}(\Omega)$ independent of $w \in L^{p}(\Omega)$. In particular, we can find a constant $\beta$, independent of $w$, such that

$$
\beta \varphi_{1}(x)<u(x) \text { in } \Omega \text {. }
$$

Furthermore, we can choose a positive constant $\alpha$ so large that

$$
\left\{\begin{array}{l}
\alpha+\beta>s_{+}, \\
\alpha \varphi_{1}(x)+\beta \varphi_{1}(x)>z(x) \quad \text { in } \Omega .
\end{array}\right.
$$

Indeed, it suffices to note (see [16, Proposition 3.8]) that there exists a positive constant $\gamma$ such that

$$
-\gamma \varphi_{1}(x) \leq z(x) \leq \gamma \varphi_{1}(x) \text { in } \Omega,
$$

since $B z=0$ on $\partial \Omega$.

Step 2: Now, by the orthogonal decomposition (3.4) of $L^{p}(\Omega)$ we can consider an operator equation

$$
v=\left(A-\lambda_{1} I\right)^{-1} G\left(x, \alpha \varphi_{1}(x)+v(x)\right), \quad v \in L^{p}(\Omega),
$$

in the closed subspace $\mathcal{R}\left(A_{p}-\lambda_{1} I\right)=\mathcal{N}\left(A_{p}-\lambda_{1} I\right)^{\perp}$ of $L^{p}(\Omega)$. Then, by estimate (3.8) with

$$
w(x):=\alpha \varphi_{1}(x)+v(x), \quad \alpha>0, \quad v \in L^{p}(\Omega),
$$

it is easy to verify the following two assertions (I) and (II):

(I) The mapping $\left(A-\lambda_{1} I\right)^{-1} G\left(x, \alpha \varphi_{1}+\cdot\right): \mathcal{R}\left(A_{p}-\lambda_{1} I\right) \rightarrow \mathcal{R}\left(A_{p}-\lambda_{1} I\right)$ is compact.

(II) $\left\|\left(A-\lambda_{1} I\right)^{-1}\left(G\left(x, \alpha \varphi_{1}+v\right)\right)\right\|_{W^{2, p}(\Omega)} \leq k C|\Omega|^{1 / p}$ for all $v \in \mathcal{R}\left(A_{p}-\lambda_{1} I\right)$.

Therefore, by applying Schauder's fixed point theorem to our situation we obtain that the operator equation (3.11) has a fixed point $v \in \mathcal{R}\left(A_{p}-\lambda_{1} I\right) \cap B\left(0, k C|\Omega|^{1 / p}\right)$, where $B(0, r)$ denotes the closed ball of radius $r$ about the origin 0 in $L^{p}(\Omega)$. In other words, there exists a solution $v \in W^{2, p}(\Omega) \cap \mathcal{R}\left(A_{p}-\lambda_{1} I\right)$ of the semilinear elliptic boundary value problem

$$
\begin{cases}A v-\lambda_{1} v=G\left(x, \alpha \varphi_{1}(x)+v(x)\right) & \text { in } \Omega, \\ B v=0 & \text { on } \partial \Omega .\end{cases}
$$

Step 3: If we let

$$
u_{+}:=\alpha \varphi_{1}+v \in W^{2, p}(\Omega),
$$

then we have, by inequality (3.9) with $u:=v$ and inequality (3.10),

$$
u_{+}(x)=\alpha \varphi_{1}(x)+v(x)>\alpha \varphi_{1}(x)+\beta \varphi_{1}(x)>z(x) \text { in } \Omega,
$$

and, by condition $\left(A_{+}\right)$,

$$
\begin{aligned}
u_{+}(x) & =\alpha \varphi_{1}(x)+v(x)>\alpha \varphi_{1}(x)+\beta \varphi_{1}(x)>s_{+} \varphi_{1}(x) \quad \text { in } \Omega \\
& \Longrightarrow \\
G\left(x, u_{+}(x)\right) & =g_{+}\left(x, u_{+}(x)\right)-\left(\int_{\Omega} g_{+}\left(y, u_{+}(y)\right) \varphi_{1}(y) d y\right) \varphi_{1}(x) \geq g_{+}\left(x, u_{+}(x)\right) \\
& \geq f\left(x, u_{+}(x)\right) \quad \text { in } \Omega .
\end{aligned}
$$


Hence, by assertion (3.12) it follows that

$$
\left(A-\lambda_{1}\right) u_{+}=\left(A-\lambda_{1}\right) v=G\left(x, u_{+}(x)\right) \geq f\left(x, u_{+}(x)\right) \text { in } \Omega,
$$

and further that

$$
B u_{+}=\alpha B \varphi_{1}+B v=0 \quad \text { on } \partial \Omega .
$$

Summing up, we have constructed a supersolution $u_{+} \in W^{2, p}(\Omega)$ of problem (3.1) satisfying condition (3.2). The proof of Lemma 3.2 is now complete.

The next theorem gives sufficient conditions in order that problem (3.1) with linear part at resonance have at least one solution (cf. [9, Theorem 2.5]):

Theorem 3.3 Assume that the nonlinear term $f(x, \xi)$ satisfies the regularity condition (F.1) and conditions $\left(A_{+}\right)$and $\left(A_{-}\right)$. Then there exists at least one solution $u \in W^{2, p}(\Omega), N<p<\infty$, of problem (3.1).

Pro of. First, we take a function $z \in C^{2}(\Omega) \cap C^{1}(\bar{\Omega})$ such that

$$
B z=0 \quad \text { on } \partial \Omega .
$$

Then, by using Lemma 3.2 we can find a supersolution $u_{+} \in W^{2, p}(\Omega)$ of problem (3.1) which satisfies condition (3.2) and a subsolution $u_{-} \in W^{2, p}(\Omega)$ of problem (3.1) which satisfies condition (3.3). Therefore, the conclusion of Theorem 3.3 follows from an application of Theorem 1.1.

The next corollary gives a necessary and sufficient condition in order that problem (3.1) with linear part at resonance have at least one solution (cf. [9, Corollary 2.10]):

Corollary 3.4 Assume that the nonlinear term $f(x, \xi)$ satisfies the regularity condition $(F .1)$ and the condition

$$
\frac{\partial f}{\partial \xi}(x, \xi) \leq 0, \quad(x, \xi) \in \bar{\Omega} \times \mathbf{R} .
$$

Then there exists at least one solution $u \in W^{2, p}(\Omega), N<p<\infty$, of problem (3.1) if and only if there exists a function $u_{0} \in W^{2, p}(\Omega), N<p<\infty$, which satisfies the conditions

$$
\begin{aligned}
& B u_{0}=0 \quad \text { on } \partial \Omega, \\
& \int_{\Omega} f\left(x, u_{0}(x)\right) \varphi_{1}(x) d x=0 .
\end{aligned}
$$

Pro o f. The proof is divided into two steps.

(1) It is clear that the existence of a function $u_{0}(x)$ is necessary.

(2) Conversely, we assume that there exists a function $u_{0} \in W^{2, p}(\Omega), N<p<\infty$, which satisfies conditions (3.13) and (3.14). We verify that the function $f(x, \xi)$ satisfies both conditions $\left(A_{+}\right)$and $\left(A_{-}\right)$.

By [16, Proposition 3.8], we can find constants $s_{+}$and $s_{-}$such that

$$
s_{-} \varphi_{1}(x)<u_{0}(x)<s_{+} \varphi_{1}(x) \text { in } \Omega .
$$

If we let

$$
g_{ \pm}(x):=f\left(x, u_{0}(x)\right),
$$

then it follows that we have, for all $u>s_{+} \varphi_{1}$ in $\Omega$,

$$
\begin{aligned}
& f(x, u(x)) \leq f\left(x, u_{0}(x)\right)=g_{+}(x), \quad x \in \Omega, \\
& \int_{\Omega} g_{+}(x) \varphi_{1}(x) d x=\int_{\Omega} f\left(x, u_{0}(x)\right) \varphi_{1}(x) d x=0,
\end{aligned}
$$


since the function $f(x, \xi)$ is decreasing in $\xi$, for each $x \in \Omega$.

Similarly, we have, for all $u<s_{-} \varphi_{1}$ in $\Omega$,

$$
\begin{aligned}
& f(x, u(x)) \geq f\left(x, u_{0}(x)\right)=g_{-}(x), \quad x \in \Omega, \\
& \int_{\Omega} g_{-}(x) \varphi_{1}(x) d x=\int_{\Omega} f\left(x, u_{0}(x)\right) \varphi_{1}(x) d x=0 .
\end{aligned}
$$

Therefore, the existence of a solution $u(x)$ of problem (3.1) follows from an application of Theorem 3.3.

\subsection{End of proof of Theorem 1.3}

First, by the mean value theorem it is easy to see that condition (C) implies condition (B).

Secondly, we show that condition (B) implies condition (A). We have only to consider the case where $\xi>0$.

If condition (B) is satisfied for $\xi>0$, then we can find a large positive number $s_{0}$ and a continuous function $g(x)$ on $\bar{\Omega}$ such that

$$
\begin{aligned}
& g(x)>0, \quad x \in \bar{\Omega}, \\
& \frac{f(x, \xi)}{\xi}+g(x)<\lambda_{1}, \quad \xi>s_{0}, x \in \bar{\Omega} .
\end{aligned}
$$

Hence we have, for all $\xi>s_{0}$,

$$
f(x, \xi)-\lambda_{1} \xi<-\xi g(x) \leq-g(x), \quad x \in \bar{\Omega},
$$

and

$$
\int_{\Omega}(-g(x)) \varphi_{1}(x) d x<0 .
$$

This verifies condition (A) for $\xi>0$ with $h(x):=-g(x)$.

Thirdly, it should be noticed that condition (A) is just a restatement of conditions $\left(B_{ \pm}\right)$.

Therefore, the desired assertion of Theorem 1.3 follows by combining Lemma 3.1 and Theorem 3.3.

\section{Proof of Theorem 1.5}

In this section we prove our existence theorem of solutions of problem (1.5) depending on a parameter (Theorem 1.5). To do this, we construct sub- and supersolutions of problem (1.5) just as in Subsection 3.1. The essential step in the proof is Theorem 4.3 in which we give sufficient conditions in order that $t_{0}>-\infty$.

\subsection{Equations depending on a parameter}

In this subsection we consider problem (1.5) depending on a parameter $t$. We recall that a solution of problem (1.5) is a function $u \in W^{2, p}(\Omega), N<p<\infty$, which satisfies the conditions

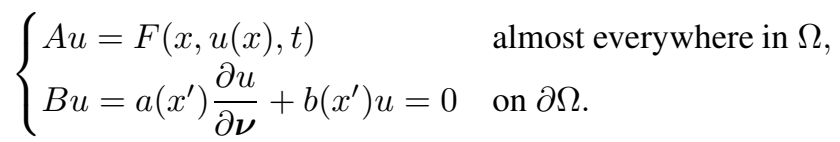

Similarly, a supersolution of problem (1.5) is a function $\psi \in W^{2, p}(\Omega), N<p<\infty$, which satisfies the conditions

$$
\begin{cases}A \psi \geq F(x, \psi(x), t) & \text { almost everywhere in } \Omega, \\ B \psi \geq 0 & \text { on } \partial \Omega,\end{cases}
$$


and a subsolution of problem (1.5) is a function $\phi \in W^{2, p}(\Omega), N<p<\infty$, which satisfies the conditions

$$
\begin{cases}A \phi \leq F(x, \phi(x), t) & \text { almost everywhere in } \Omega, \\ B \phi \leq 0 & \text { on } \partial \Omega .\end{cases}
$$

The next existence theorem of solutions of problem (1.5) is a generalization of Theorem 1.1 to the parameter dependence case:

Theorem 4.1 Assume that $F(x, \xi, t)$ satisfies the regularity condition (F.2). If $\psi(x)$ and $\phi(x)$ are respectively super-and subsolutions of problem (1.5) satisfying the condition

$$
\phi(x) \leq \psi(x) \quad \text { on } \bar{\Omega},
$$

then there exists a solution $u \in W^{2, p}(\Omega), N<p<\infty$, of problem (1.5) such that $\phi(x) \leq u(x) \leq \psi(x)$ on $\bar{\Omega}$.

We give simple conditions on the nonlinear term $F(x, \xi, t)$ in order that there exist a number $t_{0} \in[-\infty,+\infty]$ such that problem (1.5) has no (classical) solution for $t>t_{0}$ and at least one solution for $t<t_{0}$ (cf. [9, Theorem 3.4]):

Theorem 4.2 Assume that the function $F(x, \xi, t)$ satisfies the condition

$$
\frac{\partial F}{\partial t}(x, \xi, t) \geq 0, \quad(x, \xi, t) \in \bar{\Omega} \times \mathbf{R} \times \mathbf{R},
$$

and further that the function $f(x, \xi, t)$, defined by the formula

$$
f(x, \xi, t):=F(x, \xi, t)-\lambda_{1} \xi, \quad(x, \xi, t) \in \bar{\Omega} \times \mathbf{R} \times \mathbf{R},
$$

satisfies condition $\left(A_{-}\right)$. Then there exists a number $t_{0} \in[-\infty,+\infty]$ such that problem (1.5) has no (classical) solution for $t>t_{0}$ and at least one solution $u \in W^{2, p}(\Omega), N<p<\infty$, for $t<t_{0}$.

Proof. Assume that problem (1.5) has a solution $v \in W^{2, p}(\Omega), N<p<\infty$, for some $t=c$. By condition (4.1), it follows that the function $u_{+}(x):=v(x)$ is a supersolution of problem (1.5) for all $t \leq c$. Indeed, we have the assertions

$$
\begin{cases}A u_{+}=F\left(x, u_{+}(x), c\right) \geq F\left(x, u_{+}(x), t\right) & \text { almost everywhere in } \Omega, \\ B u_{+}=0 & \text { on } \partial \Omega .\end{cases}
$$

Moreover, by using Lemma 3.2 we can find a subsolution $u_{-} \in W^{2, p}(\Omega), N<p<\infty$, of problem (1.5) which satisfies the condition $u_{-}(x)<u_{+}(x)$ in $\Omega$. Namely, we have the assertions

$$
\begin{cases}A u_{-} \leq F\left(x, u_{-}(x), t\right) & \text { almost everywhere in } \Omega, \\ u_{-}<u_{+} & \text {in } \Omega, \\ B u_{-}=0 & \text { on } \partial \Omega .\end{cases}
$$

Hence it follows from an application of Theorem 4.1 that problem (1.5) has a solution $u \in W^{2, p}(\Omega), N<p<$ $\infty$, for all $t \leq c$. Therefore, we have only to take

$$
t_{0}:=\sup \{t \in \mathbf{R}: \text { problem (1.5) has a solution for } t\} .
$$

The proof of Theorem 4.2 is complete.

Furthermore, we give some conditions which insure that $t_{0}>-\infty$ and $t_{0}<+\infty$. The next theorem gives sufficient conditions in order that $t_{0}>-\infty$ (cf. [9, Theorem 3.5]):

Theorem 4.3 In addition to the conditions of Theorem 4.2, we assume that there is a function $h \in C(\bar{\Omega})$ satisfying the condition $h(x)>0$ in $\Omega$ and that there exist numbers $0 \leq m_{0}<m_{1}$ and $t_{1}$ such that

$$
\frac{\partial F}{\partial t}(x, \xi, t) \geq h(x) \geq 0, \quad x \in \bar{\Omega}, m_{0} \leq \xi \leq m_{1}, t \leq t_{1} .
$$

Then there exists a number $t \in \mathbf{R}$ such that problem (1.5) has at least one solution $u \in W^{2, p}(\Omega)$ with $N<p<$ $\infty$. In particular, it follows that $t_{0}>-\infty$. 
Proof. The proof of Theorem 4.3 is divided into three steps.

Step 1: First, by arguing just as in the proof of Theorem 4.2 with $u_{+}:=0$ we can construct a subsolution $u_{-} \in W^{2, p}(\Omega), N<p<\infty$, of problem (1.5); more precisely, we can construct a function $u_{-} \in W^{2, p}(\Omega)$ (see assertion (4.2)) such that

$$
\begin{cases}A u_{-} \leq F\left(x, u_{-}(x), t\right) & \text { in } \Omega \\ u_{-}<0 & \text { in } \Omega \\ B u_{-}=0 & \text { on } \partial \Omega .\end{cases}
$$

Step 2: Secondly, we construct a supersolution $u_{+} \in W^{2, p}(\Omega), N<p<\infty$, of problem (1.5) for some $t$. To do this, we may assume that $t_{1}=0$. If we let

$$
f(x, \xi):=F\left(x, \xi, t_{1}\right)=F(x, \xi, 0),
$$

then we have, by condition (4.3),

$$
F(x, \xi, t)-t h(x) \leq f(x, \xi), \quad x \in \bar{\Omega}, m_{0} \leq \xi \leq m_{1}, t \leq 0 .
$$

Hence it suffices to find a function $u_{+}(x)$ such that

$$
\begin{cases}A u_{+} \geq t h(x)+f\left(x, u_{+}(x)\right) & \text { in } \Omega \\ B u_{+} \geq 0 & \text { on } \partial \Omega\end{cases}
$$

and that

$$
m_{0} \leq u_{+}(x) \leq m_{1} \quad \text { in } \Omega
$$

We let

$$
\omega:=\sup _{\substack{x \in \bar{\Omega} \\ m_{0} \leq \xi \leq m_{1}}} f(x, \xi) .
$$

(i) The case where $\omega \leq 0$ : In this case, we can take $u_{+}(x) \equiv m_{0}$ and $t=0$ to satisfy conditions (4.4). Indeed, we have the assertions

$$
\begin{cases}A u_{+}=c(x) m_{0} \geq 0 \geq \omega \geq f\left(x, u_{+}(x)\right) & \text { in } \Omega, \\ B u_{+}=b\left(x^{\prime}\right) m_{0} \geq 0 & \text { on } \partial \Omega .\end{cases}
$$

(ii) The case where $0<\omega<+\infty$ : Let $w(x)$ be an arbitrary function in $W^{2, p}(\Omega)$, with $N<p<\infty$, which satisfies the boundary condition

$$
B w=0 \quad \text { on } \partial \Omega .
$$

By using [17, Theorem 1.1] and Sobolev's imbedding theorem, we obtain that, for some positive constant $C$,

$$
\|w\|_{W^{1, \infty}(\Omega)} \leq C\|A w\|_{L^{p}(\Omega)}
$$

and further that

$$
w \in W^{2, p}(\Omega) \subset C^{1}(\bar{\Omega}) \subset W^{1, \infty}(\Omega), \quad N<p<\infty .
$$

We take two open subsets $\Omega_{1}$ and $\Omega_{2}$ of $\Omega$ such that

$$
\left\{\begin{array}{l}
\Omega_{1} \Subset \Omega_{2} \Subset \Omega, \\
h(x)>0 \text { on } \overline{\Omega_{2}}, \\
\left|\Omega \backslash \Omega_{1}\right|<\left(\frac{m_{1}-m_{0}}{\omega C}\right)^{p}
\end{array}\right.
$$


and let $H(x)$ be a smooth function on $\bar{\Omega}$ which satisfies the conditions

$$
\begin{cases}0 \leq H(x) \leq \omega & \text { in } \Omega, \\ H(x)=\omega & \text { in } \Omega \backslash \Omega_{2}, \\ H(x)=0 & \text { in } \Omega_{1} .\end{cases}
$$

Moreover, if we take a large negative number $t$ so that

$$
\omega+\operatorname{th}(x)<0 \quad \text { in } \Omega_{2},
$$

then it is easy to see that

$$
H(x) \geq \omega+t h(x) \quad \text { in } \Omega .
$$

It should be emphasized that this $t$ will be the value of $t$ for which problem (1.5) has at least one solution $u \in W^{2, p}(\Omega)$ with $N<p<\infty$.

Now we let $v \in W^{2, p}(\Omega), N<p<\infty$, be the unique solution of the linear problem

$$
\begin{cases}A v=H(x) & \text { in } \Omega, \\ B v=0 & \text { on } \partial \Omega .\end{cases}
$$

Then it follows from an application of the maximum principle ( [17, Proposition 3.12]) that

$$
v(x)>0 \text { in } \Omega .
$$

On the other hand, by applying inequality (4.6) with $w:=v$ and conditions (4.8) and (4.7) we obtain that

$$
\begin{aligned}
v(x) & \leq\|v\|_{L^{\infty}(\Omega)} \leq C\|A v\|_{L^{p}(\Omega)}=C\|H\|_{L^{p}(\Omega)}=C\left(\int_{\Omega \backslash \Omega_{1}} H(x)^{p} d x\right)^{1 / p} \\
& \leq \omega C\left|\Omega \backslash \Omega_{1}\right|^{1 / p}<m_{1}-m_{0}, \quad x \in \bar{\Omega} .
\end{aligned}
$$

If we let

$$
u_{+}(x):=v(x)+m_{0}, \quad x \in \Omega,
$$

then it follows that the function $u_{+}(x)$ satisfies condition (4.5). Indeed, we have, by assertions (4.11) and (4.12),

$$
m_{0}<u_{+}(x)=m_{0}+v(x)<m_{1} \quad \text { in } \Omega,
$$

and so

$$
f\left(x, u_{+}(x)\right) \leq \omega=\sup _{\substack{x \in \bar{\Omega} \\ m_{0} \leq \xi \leq m_{1}}} f(x, \xi) \text { in } \Omega .
$$

Furthermore, by combining formula (4.10), inequalities (4.9) and (4.13) we obtain that

$$
\begin{aligned}
A u_{+} & =A v+c(x) m_{0} \geq A v=H(x) \geq t h(x)+\omega \\
& \geq t h(x)+f\left(x, u_{+}(x)\right) \quad \text { in } \Omega,
\end{aligned}
$$

and further that

$$
B u_{+}=B v+B m_{0}=b\left(x^{\prime}\right) m_{0} \geq 0 \quad \text { on } \partial \Omega .
$$

Summing up, we have constructed the desired supersolution $u_{+} \in W^{2, p}(\Omega)$ of problem (1.5).

Step 3: Therefore, it follows from an application of Theorem 4.1 that problem (1.5) has a solution $u \in$ $W^{2, p}(\Omega)$ with $N<p<\infty$.

The proof of Theorem 4.3 is now complete. 
The next theorem gives some conditions under which $t_{0}<+\infty$ (see [9, Theorem 3.8]):

Theorem 4.4 Assume that the following two conditions (a) and (b) are satisfied:

(a) If $u \in W^{2, p}(\Omega), N<p<\infty$, is a solution of problem (1.5), then we have the assertion

$$
u(x) \geq c(t) \varphi_{1}(x), \quad x \in \Omega,
$$

where $c(t)$ is a function such that $c(t) \rightarrow+\infty$ as $t \rightarrow+\infty$.

(b) There exist numbers $s_{1} \in[-\infty,+\infty)$ and $t_{2} \in[-\infty,+\infty)$ such that we have, for all $u>s_{1} \varphi_{1}$ and $t>t_{2}$,

$$
\int_{\Omega}\left(F(x, u(x), t)-\lambda_{1} u(x)\right) \varphi_{1}(x) d x>0 .
$$

Then there exists a number $t_{1} \in[-\infty,+\infty)$ such that problem (1.5) has no solution for $t>t_{1}$.

The next proposition gives verifiable conditions under which condition (b) of Theorem 4.4 is satisfied (see [9, Proposition 3.9]):

Proposition 4.5 Assume that the function $F(x, \xi, t)$ satisfies the condition

$$
\frac{\partial F}{\partial t}(x, \xi, t) \geq 0, \quad(x, \xi, t) \in \bar{\Omega} \times \mathbf{R} \times \mathbf{R},
$$

and further that any of the following three conditions (b.1), (b.2) and (b.3) holds true for some $t=t_{1}$ :

(b.1) There exists a continuous function $h(x)$ on $\bar{\Omega}$ such that

$$
\begin{aligned}
& \int_{\Omega} h(x) \varphi_{1}(x) d x>0, \\
& \liminf _{\xi \rightarrow+\infty}\left(F\left(x, \xi, t_{1}\right)-\lambda_{1} \xi\right)>h(x), \quad x \in \bar{\Omega},
\end{aligned}
$$

where $\liminf _{\xi \rightarrow+\infty}$ is assumed to be uniform for $x \in \bar{\Omega}$.

(b.2) We have the condition

$$
\liminf _{\xi \rightarrow+\infty} \frac{F\left(x, \xi, t_{1}\right)}{\xi}>\lambda_{1},
$$

where $\liminf _{\xi \rightarrow+\infty}$ is assumed to be uniform for $x \in \bar{\Omega}$.

(b.3) We have the condition

$$
\liminf _{\xi \rightarrow+\infty} \frac{\partial F}{\partial \xi}\left(x, \xi, t_{1}\right)>\lambda_{1},
$$

where $\liminf _{\xi \rightarrow+\infty}$ is assumed to be uniform for $x \in \bar{\Omega}$.

Then it follows that condition (b) of Theorem 4.4 is satisfied.

The next proposition gives verifiable conditions under which condition (a) of Theorem 4.4 is satisfied (see [9, Proposition 3.10]):

Proposition 4.6 Assume that the following two conditions (a.1) and (a.2) are satisfied:

(a.1) There exist constants $\gamma<\lambda_{1}, k$ and $t_{1}$ such that

$$
F(x, \xi, t)-\gamma \xi \geq k, \quad x \in \bar{\Omega}, \xi \in \mathbf{R}, t \geq t_{1} .
$$


(a.2) For every $m \in \mathbf{R}$, there exists a non-negative, continuous function $h_{m}(x)$ on $\bar{\Omega}$, depending on $m$, such that

$$
\frac{\partial F}{\partial t}(x, \xi, t) \geq h_{m}(x) \geq 0, \quad x \in \bar{\Omega}, \xi \geq m, t \geq t_{1}
$$

If $u \in W^{2, p}(\Omega), N<p<\infty$, is a solution of problem (1.5) for some $t \geq t_{1}$, then we have the inequality

$$
u(x) \geq(a t+b) \varphi_{1}(x), \quad x \in \bar{\Omega}
$$

for some constants $a>0$ and $b \in \mathbf{R}$ independent of $u$.

Remark 4.7 The above condition (4.15) insures condition (4.14) with $c(t):=a t+b$.

\subsection{End of proof of Theorem 1.5}

The proof of Theorem 1.5 is divided into three steps.

Step 1: First, we prove that $t_{0}>-\infty$ : By condition (D.1), it follows that

$$
\frac{\partial F}{\partial t}(x, \xi, t)>0, \quad x \in \Omega, \xi \geq m, t \in \mathbf{R} .
$$

By condition (D.2), we can prove that, for every $t$, the function

$$
f(x, \xi, t):=F(x, \xi, t)-\lambda_{1} \xi
$$

satisfies condition $\left(A_{-}\right)$just as in the proof of Theorem 1.3 (see Subsection 3.2). Therefore, it follows from an application of Theorem 4.2 that there exists a number $t_{0} \in[-\infty,+\infty]$ such that problem (1.5) has at least one solution $u \in W^{2, p}(\Omega), N<p<\infty$, for $t<t_{0}$, but it has no solution for $t>t_{0}$.

Step 2: Secondly, by condition (D.1) it follows from an application of Theorem 4.3 that $t_{0}>-\infty$.

Step 3: Thirdly, we prove that $t_{0}<+\infty$ : To do this, we apply Theorem 4.4.

Step 3-1: The condition (b) of Theorem 4.4 follows by combining conditions (D.3) and (D.1) with $m:=m_{1}$. Indeed, we have, for all $t_{2}>t_{1}$,

$$
\begin{aligned}
F\left(x, \xi, t_{2}\right)-\lambda_{1} \xi & =\left(F\left(x, \xi, t_{1}\right)-\lambda_{1} \xi\right)+\left(F\left(x, \xi, t_{2}\right)-F\left(x, \xi, t_{1}\right)\right) \\
& >c+h_{m_{1}}(x)\left(t_{2}-t_{1}\right), \quad \xi>m_{1} .
\end{aligned}
$$

This proves that

$$
\liminf _{\xi \rightarrow+\infty}\left(F\left(x, \xi, t_{2}\right)-\lambda_{1} \xi\right) \geq c+h_{m_{1}}(x)\left(t_{2}-t_{1}\right), \quad x \in \bar{\Omega} .
$$

If we take a number $t_{2}$ so large that

$$
t_{2}>t_{1}-\frac{c \int_{\Omega} \varphi_{1}(x) d x}{2 \int_{\Omega} h_{m_{1}}(x) \varphi_{1}(x) d x}
$$

and let

$$
g(x):=\frac{c}{2}+h_{m_{1}}(x)\left(t_{2}-t_{1}\right), \quad x \in \bar{\Omega},
$$

then we obtain from inequality (4.16) that

$$
\liminf _{\xi \rightarrow+\infty}\left(F\left(x, \xi, t_{2}\right)-\lambda_{1} \xi\right) \geq c+h_{m_{1}}(x)\left(t_{2}-t_{1}\right)>\frac{c}{2}+h_{m_{1}}(x)\left(t_{2}-t_{1}\right)=g(x), \quad x \in \bar{\Omega},
$$

and further from inequality (4.17) that

$$
\int_{\Omega} g(x) \varphi_{1}(x) d x=\frac{c}{2} \int_{\Omega} \varphi_{1}(x) d x+\left(t_{2}-t_{1}\right) \int_{\Omega} h_{m_{1}}(x) \varphi_{1}(x) d x>0 .
$$


This verifies condition (b.1) of Proposition 4.5 with $h(x):=g(x)$.

Therefore, the desired condition (b) of Theorem 4.4 follows an application of Proposition 4.5.

Step 3-2: On the other hand, we make use of Proposition 4.6 to verify the desired condition (a) of Theorem 4.4.

(1) By using condition (D.2), we can find a constant $\gamma<\lambda_{1}$ and a negative number $m_{0}$ such that we have, for all $\xi<m_{0}$,

$$
\frac{F\left(x, \xi, t_{1}\right)}{\xi}<\gamma, \quad x \in \bar{\Omega} .
$$

More precisely, there exists a constant $c_{1}$ such that

$$
F\left(x, \xi, t_{1}\right)-\gamma \xi>c_{1}, \quad \xi<m_{0}, x \in \bar{\Omega} .
$$

In view of condition (D.1) (with $m<\xi<m_{0}$ ), this proves that

$$
F(x, \xi, t)-\gamma \xi \geq F\left(x, \xi, t_{1}\right)-\gamma \xi>c_{1}, \quad t \geq t_{1}, \xi<m_{0}, x \in \bar{\Omega}
$$

(2) Furthermore, by condition (D.3) it follows that

$$
\begin{gathered}
F(x, \xi, t)-\gamma \xi \geq F\left(x, \xi, t_{1}\right)-\gamma \xi=\left(F\left(x, \xi, t_{1}\right)-\lambda_{1} \xi\right)+\left(\lambda_{1}-\gamma\right) \xi>c+\left(\lambda_{1}-\gamma\right) \xi \\
>c_{2}:=c+\left(\lambda_{1}-\gamma\right) m_{1}, \quad t \geq t_{1}, \xi>m_{1}, x \in \bar{\Omega} .
\end{gathered}
$$

(3) It is obvious that we have, for some constant $c_{3}$,

$$
F(x, \xi, t)-\gamma \xi \geq F\left(x, \xi, t_{1}\right)-\gamma \xi>c_{3}, \quad t \geq t_{1}, m_{0} \leq \xi \leq m_{1}, x \in \bar{\Omega}
$$

Therefore, by combining inequalities (4.18), (4.19) and (4.20) we obtain that condition (a.1) of Proposition 4.6 holds true with $k:=\min \left(c_{1}, c_{2}, c_{3}\right)$.

(4) Finally, we remark that condition (a.2) of Proposition 4.6 is a part of condition (D.1).

The proof of Theorem 1.5 is now complete.

\subsection{Proof of Corollary 1.7}

In order to prove Corollary 1.7, we have only to verify all the conditions (D.1), (D.2) and (D.3) of Theorem 1.5 for the function $F(x, \xi, t)$ given by formula (1.6). Corollary 1.7 can be proved just as in the proof of KazdanWarner [9, Corollary 3.12].

\section{Existence of positive solutions}

The purpose of this final section is to find a positive solution of the following semilinear elliptic eigenvalue problem: Given a function $f(x, \xi)$ defined on $\bar{\Omega} \times \mathbf{R}$, find a positive function $u(x)$ in $\Omega$ such that

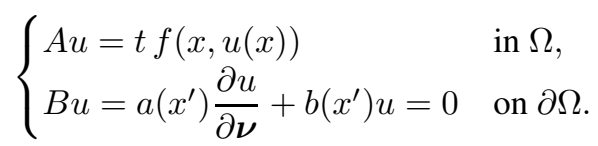

The next existence theorem of positive solutions of problem (5.1) extends Kazdan-Warner [9, Proposition 4.2] to the degenerate case (cf. [6, Theorem 2.2]; [15, Theorem 4]):

Theorem 5.1 Assume that the nonlinear term $f(x, \xi)$ satisfies the regularity condition (F.1) and further that

$$
f(x, \xi)>0, \quad(x, \xi) \in \Omega \times \mathbf{R} .
$$

Then there exists a number $t_{0}, 0<t_{0} \leq+\infty$, such that problem (5.1) has a positive solution for $0<t<t_{0}$, but it has no positive solution for $t \leq 0$ and $t>t_{0}$. Moreover, we have the following two assertions (i) and (ii): 
(i) If we have the condition

$$
\liminf _{\xi \rightarrow+\infty} \frac{f(x, \xi)}{\xi}>0,
$$

where $\liminf _{\xi \rightarrow+\infty}$ is assumed to be uniform for $x \in \bar{\Omega}$, then it follows that $0<t_{0}<+\infty$.

(ii) If we have the condition

$$
\lim _{\xi \rightarrow+\infty} \frac{f(x, \xi)}{\xi}=0
$$

where $\lim _{\xi \rightarrow+\infty}$ is assumed to be uniform for $x \in \bar{\Omega}$, then it follows that $t_{0}=+\infty$. Namely, problem (5.1) has a positive solution for all $t>0$.

Theorem 5.1 can be proved just as in the proof of Kazdan-Warner [9, Proposition 4.2].

Acknowledgements The author is grateful to the referees for many valuable suggestions which improved the presentation of this paper and for informing him of some additional references. This research is partially supported by Grant-in-Aid for General Scientific Research (No. 19540162), Ministry of Education, Culture, Sports, Science and Technology, Japan.

\section{References}

[1] R. A. Adams and J. J. F. Fournier, Sobolev spaces, second edition (Academic Press, Amsterdam Heidelberg New York Oxford, 2003).

[2] H. Amann, Fixed point equations and nonlinear eigenvalue problems in ordered Banach spaces, SIAM Rev. 18, 620-709 (1976).

[3] H. Amann and M. G. Crandall, On some existence theorems for semi-linear elliptic equations, Indiana Univ. Math. J. 27 (1978), 779-790.

[4] J.-M. Bony, Principe du maximum dans les espaces de Sobolev, C. R. Acad. Sc. Paris 265, 333-336 (1967).

[5] K. C. Chang, Methods in nonlinear analysis (Springer-Verlag, Berlin Heidelberg New York, 2005).

[6] D. G. de Figueiredo, Positive solutions of semilinear elliptic problems, Lecture Notes in Mathematics, No. 957 (Springer-Verlag, Berlin Heidelberg New York, 1982), 34-87.

[7] S. Fučík, Solvability of nonlinear equations and boundary value problems (D. Reidel Publishing Company, Dordrecht Boston London, 1980).

[8] L. Hörmander, The analysis of linear partial differential operators III (Springer-Verlag, Berlin Heidelberg New York Tokyo, 1985).

[9] J. L. Kazdan and F. W. Warner, Remarks on some quasilinear elliptic equations, Comm. Pure Appl. Math. 28, 567-597 (1975).

[10] T. Runst, On the existence of solutions of nonlinear boundary value problems at resonance in Sobolev spaces of fractional order, Hiroshima Math. J. 29, 313-325 (1999).

[11] T. Runst and Y. Il'yasov, On equations of type $A u=g(x, u, \nabla u)$ with degenerate and nonlinear boundary conditions, Tsukuba J. Math. 23, 505-528 (1999).

[12] T. Runst and W. Sickel, Sobolev spaces of fractional order, Nemytskij operators, and nonlinear partial differential equations (Walter de Gruyter, Berlin New York, 1996).

[13] D. H. Sattinger, Topics in stability and bifurcation theory, Lecture Notes in Mathematics, No. 309 (Springer-Verlag, Berlin New York, 1973).

[14] K. Taira, Existence and uniqueness theorems for semilinear elliptic boundary value problems, Adv. Differential Equations, 2, 509-534 (1997).

[15] K. Taira, Bifurcation theory for semilinear elliptic boundary value problems, Hiroshima Math. J. 28, 261-308 (1998).

[16] K. Taira, Diffusive logistic equations with degenerate boundary conditions, Mediterr. J. Math. 1, 315-365 (2004).

[17] K. Taira, Degenerate elliptic boundary value problems with indefinite weights, Mediterr. J. Math. 5, 133-162 (2008).

[18] G. M. Troianiello, Elliptic differential equations and obstacle problems (Plenum Press, New York, 1987).

[19] E. Zeidler, Nonlinear functional analysis and its applications I (Springer-Verlag, New York Berlin Heidelberg, 1986). 